\title{
Tunable Single-Longitudinal-Mode Fiber Optical Parametric Oscillator with Saturable-Absorber-Based Auto-Tracking Filter
}

\author{
Sigang Yang, Kim K. Y. Cheung, Xing Xu, Yue Zhou, and Kenneth K. Y. Wong* \\ Photonic Systems Research Laboratory, Department of Electrical and Electronic Engineering, \\ The University of Hong Kong, Pokfulam Road, Hong Kong. \\ *Email:kywong@eee.hku.hk
}

\begin{abstract}
A tunable single-longitudinal-mode (SLM) fiber optical parametric oscillator is demonstrated by using a subring cavity and a fiber loop mirror incorporated with a saturable absorber. It can provide dual-wavelength SLM output.

(C)2010 Optical Society of America

OCIS codes: (060.4370) Nonlinear optics, fibers; (190.4970) Parametric oscillators and amplifiers
\end{abstract}

\section{Introduction}

Fiber optical parametric oscillators (FOPO) based on four-wave mixing (FWM) occurring inside optical fibers has attracted considerable attention because it can eliminate the complicated alignment as in bulk OPO, is ready to satisfy phase matching and compatible with fiber-based devices as in optical communications [1]. To obtain enough optical parametric amplification (OPA) gain to overcome the cavity loss, usually much longer OPA gain fiber is used compared with the bulk OPO. Thus, the FOPO has many densely spaced longitudinal modes unavoidably because of its long cavity length. It is difficult to make an intracavity optical filter to select and track a single frequency [2]. Consequently, the output from the FOPO is multiple-longitudinal-mode inherently, and usually unstable with a large linewidth owing to multimode oscillation, modes competition, and hopping [3].

In this paper, we propose and demonstrate a FOPO with single-longitudinal-mode (SLM) operation. In the proposed configuration, a subring cavity is used first to broaden the longitudinal mode spacing and suppress the longitudinal modes. And then a fiber loop mirror with a saturable absorber is used as a narrow bandwidth auto-tracking optical filter. Spatial-hole burning effect is generated in the absorber when counterpropagating light waves are launched into the loop mirror. The spatial-hole burning effects lead to slight refractive index variation in the absorber. Thus a weak Bragg grating with very narrow reflection bandwidth is generated [4]. Only single longitudinal mode can oscillate when two above mentioned measures are combined. Because the narrow bandwidth filter can auto-track the oscillating wavelength, the proposed SLM FOPO is tunable.

\section{Experimental setup}

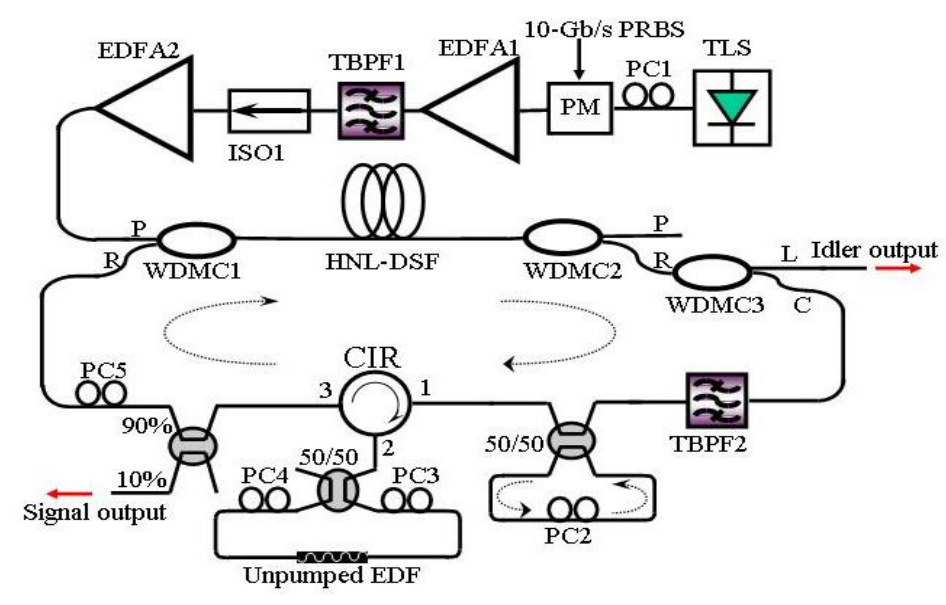

Fig.1. Schematic diagram of the single-longitudinal-mode FOPO. 
The proposed configuration and experimental setup is shown in Fig. 1. The pump is seeded by an external cavity tunable laser source (TLS) at the wavelength of $1556 \mathrm{~nm}$. To suppress SBS, the light from the TLS is first phase-modulated with $10-\mathrm{Gb} / \mathrm{s}$ pseudo-random bit sequence (PRBS) signal via a phase modulator (PM). Polarization controller PC1 aligns the pump's state of polarization (SOP) with the transmission axis of the PM. The SBS can be suppressed by up to $28 \mathrm{~dB}$. Then the pump is amplified by a two-stage configuration of EDFA, in which the first stage (EDFA1) provides small signal gain to prevent self-saturation by amplified spontaneous emission (ASE). The 0.35-nm tunable bandpass filter (TBPF1) is used to reduce amplified spontaneous emission (ASE) noise. After an isolator (ISO1) it is further amplified by the second stage (EDFA2), with a maximum average output power of $33 \mathrm{dBm}$. Then the pump is coupled into 400-m highly-nonlinear dispersion-shifted fiber (HNL-DSF) with the zero dispersion wavelength at $1554 \mathrm{~nm}$ via P-port (transmission band: $1554.89 \sim 1563.89 \mathrm{~nm}$ ) of a WDM coupler (WDMC1). The high power pump propagates through the HNL-DSF and is then rejected through P-port of another similar WDM coupler (WDMC2), while the amplified signal and idler propagate firstly through the R-port (reflection bands: $1500 \sim 1551 \mathrm{~nm}, 1567 \sim 1620 \mathrm{~nm}$ ) of WDMC2 and subsequently are split into two paths by a C/L band WDM coupler (WDMC3). Only the signal is coupled into the cavity and can oscillate. The setup provides an idler output from the $\mathrm{L}$ port of the $\mathrm{C} / \mathrm{L}$ band coupler. The signal from the $\mathrm{C}$ port of WDMC3 is filtered by a $0.35 \mathrm{~nm}$ band pass filter (TBPF2) which determines the lasing wavelength and provides the first restriction on the possible oscillating modes. The sub-ring cavity with a cavity length of about $4.1 \mathrm{~m}$ is then inserted after TBPF2. It is composed of a PC and a 50/50 coupler. Subsequently, a fiber loop mirror is introduced via a optical circulator (CIR). The loop mirror consists of a 50/50 coupler, two polarization controllers (PC3 and PC4) and a length of umpumped EDF that serves as saturable absorber. The two in-line polarization controllers (PC3 and PC4) ensure that the state of polarization of the light is properly controlled. The absorber-based fiber loop mirror forms a passive auto-tracking narrow-band optical filter. After that a 10/90 optical coupler is used to couple out $10 \%$ of signal light. PC5 is used to align the signal's SOP with the pump so as to maximize the signal gain.

\section{Experimental results and discussions}

The transmission central wavelength of TBPF2 is set to be $1542.34 \mathrm{~nm}$ at first. When the OPA gain is larger than the cavity loss, the cavity begins to oscillate. The output power of the signal is measured to be $0.6 \mathrm{dBm}$. The mode spacing is measured by a self-homodyne method, which consists of a photodetector (PD) with 3-dB bandwidth of $26 \mathrm{GHz}$ and a Mach-Zehnder interferometer that includes an optical interferometer with delay time of $3.5 \mu \mathrm{s}$ in one arm. At first, the sub-ring cavity and the loop mirror are removed, the modes spacing of the main cavity is measured

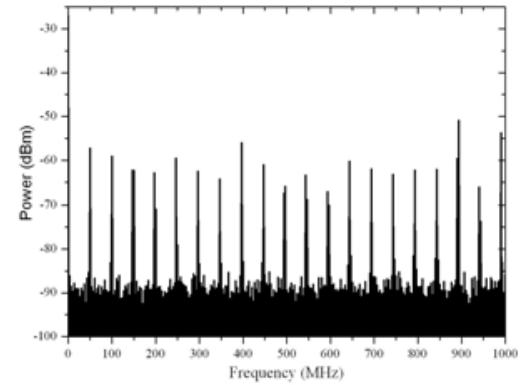

(a)

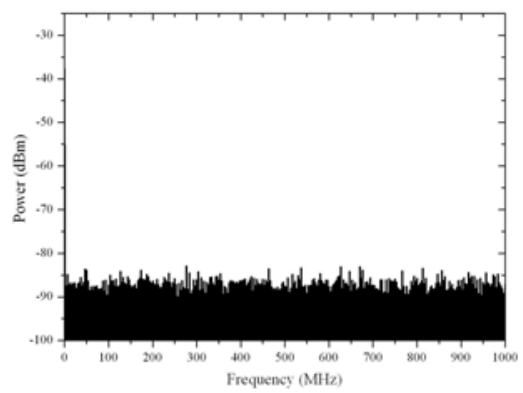

(b)

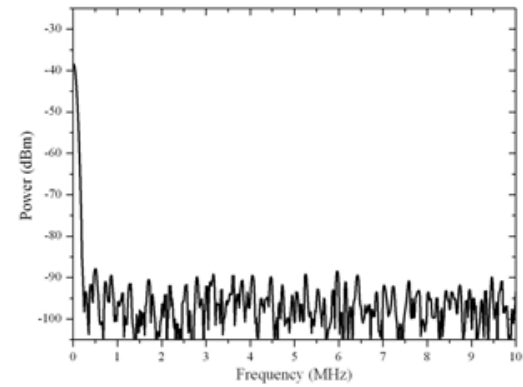

(c)

Fig.2. Measured self-homodyne spectrum: (a) with the main cavity and the sub-ring cavity connected, fiber loop mirror unconnected; (b) with all three cavities connected. (c) fine structure of the spectrum near zero frequency. 
to be only $437 \mathrm{kHz}$. And then the sub-ring cavity is inserted while the loop mirror is still removed. Fig. 2 (a) shows the measured beating signal from the electrical spectrum analyzer (ESA). It can be observed that, with the sub-ring cavity inserted, the FSR is increased from $437 \mathrm{kHz}$ to $49.5 \mathrm{MHz}$ which is determined by the FSR of the sub-ring cavity. The fundamental and higher orders beating signal can be observed clearly, while the spectrum is very noisy and unstable owing to the mode hopping. Once the fiber loop mirror is also connected, the beating signal disappears and no spike signals are observed, as shown by the detected self-homodyne frequency spectrum in Fig. 2(b). Fig. 2(c) shows the fine structure of the spectrum in Fig. 2(b) near zero frequency. Only the direct current (DC) peak can be observed while other longitudinal modes of the signal cavity are suppressed. It indicates that a SLM operation with side-mode suppression to $1 \mathrm{GHz}$ can be achieved by the proposed configuration. The idler is also measured with a self-homodyne method and the result shows it is also SLM.

Finally, the tuning feature of the proposed FOPO is investigated. When TBPF2 is tuned, the narrow bandwith filter based on the fiber loop mirror can auto-track the oscillating wavelength. The output signal from the $10 \%$ port of the 10/90 coupler and the output idler from the L port of the C/L band coupler are combined via another C/L band coupler and measured by an optical spectrum analyzer (OSA). The oscillating wavelength is tuned by adjusting the tunable optical filter (TBPF2). Fig. 3 shows its tunable characteristics of the proposed FOPO. The signal can be tuned from $1530.2 \mathrm{~nm}$ and $1540.6 \mathrm{~nm}$ and maintains the SLM operation and the corresponding idler is from 1571.4 to $1581.8 \mathrm{~nm}$. The tunable range is limited by the gain bandwidth of the proposed FOPA. Hence a tunable SLM FOPO is realized.

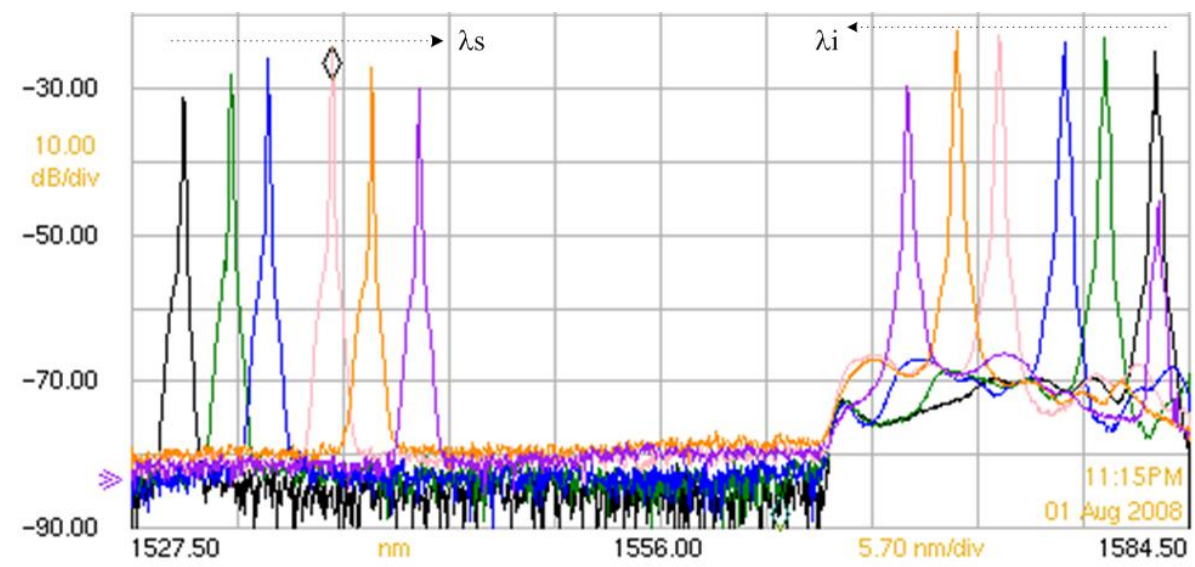

Fig.3. Overlapped optical spectra of the tunable SLM FOPO.

\section{Conclusion}

In conclusion, we have presented a tunable single-longitudinal-mode FOPO. A sub-ring cavity with short cavity length is used to broaden the longitudinal mode spacing and suppress the longitudinal modes. A fiber loop mirror with a 3.5-meter unpumped EDF is used as an auto-tracking narrow band filter, which dynamically tracks the wavelength of the FOPO. The measurement from the homodyne method shows that the FOPO provides a single-longitudinal-mode output. The FOPO can be tunable over $10 \mathrm{~nm}$ which is limited by the gain bandwidth of the FOPA.

\section{Acknowledgment}

The work was partially supported by grants from the research Grants Council of the Hong Kong Special Administrative Region, China (Project No. HKU7179/08E and HKU7183/09E). The authors would like to thank Sumitomo Electric Industries for providing HNL-DSF.

\section{References}

[1] J. E. Sharping, "Microstructure Fiber Based Optical Parametric Oscillators," IEEE J.Lightwave Technol.26,2184-2191(2008).

[2] M. E. Marhic, Fiber Optical Parametric Amplifiers, Oscillators and Related Devices, (CAMBRIDGE, 2008),Chap. 12.

[3] S. Yang, Y. Zhou, J. Li, and Kenneth K. Y. Wong, “Actively Mode-Locked Fiber Optical Parametric Oscillator,” IEEE J. Select. Topics Quant. Electron. vol.15, pp.393-398, 2009.

[4] S.J. Frisken, “ Transient Bragg reflection gratings in erbium-doped fiber amplifiers,” Opt. Lett., 17, 1776-1778 (1992). 